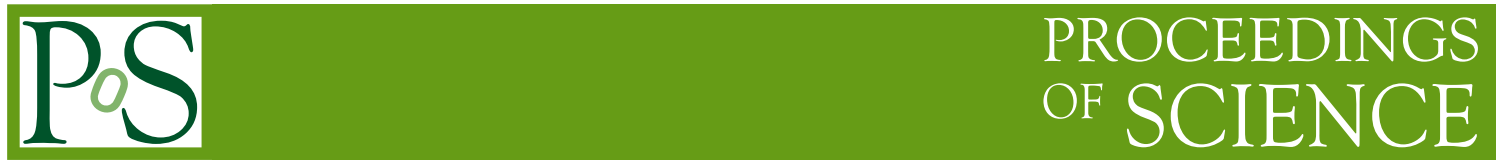

\title{
CMS Tracker Alignment and Implications on Physics Performance
}

\section{Nhan Viet TRAN*}

Johns Hopkins University, Baltimore, Maryland USA

E-mail: ntran@cern.ch

\begin{abstract}
A current overview of CMS tracker alignment is given. The overall alignment strategy is presented with an emphasis on track-based alignment as well as inputs from optical survey and the laser alignment system. Recent studies with cosmic ray data and simulation are presented, including an initial study with cosmic data involving a nearly full CMS tracker. Finally, a study is presented on the effect of alignment on physics performance, in particular, the impact on tracking. Results from current studies have been very encouraging for CMS tracker alignment in preparation for LHC startup.
\end{abstract}

2008 Physics at LHC

September 29 - 4 October 2008

Split, Croatia

${ }^{*}$ Speaker. 


\section{Introduction}

The Compact Muon Solenoid (CMS) experiment is a multipurpose detector at the LHC. The 12500 ton CMS detector consists of a $4 \mathrm{~T}$ superconducting solenoid; a muon detector; hadron calorimeter; electromagnetic calorimeter; and all-silicon tracker [1]. The tracker is made of thousands of silicon sensors whose position must be known on the order of microns. It is composed of two main parts: the silicon strip detector and the silicon pixel detector (see Fig. 1). The strip detector is composed of four main parts: the Tracker Inner Barrel (TIB), Tracker Inner Disk (TID), Tracker Outer Barrel (TOB), and Tracker Endcaps (TEC). The pixel detector is composed of two parts: the pixel endcaps (PXF) and the pixel barrels (PXB). The task of alignment is to determine module positions in order to better resolve charged particle track trajectories. Determining the precision of these module positions to the order of a few microns is essential for the discovery of new physics at CMS.

In these proceedings, a summary is given of the recent activities of CMS tracker alignment. We discuss the overall strategy employed by CMS tracker alignment. Recent alignment analyses are mentioned including first cosmic ray data from CMS as well as the most recent simulation studies. Finally, we mention the implications of tracker alignment on physics performance.
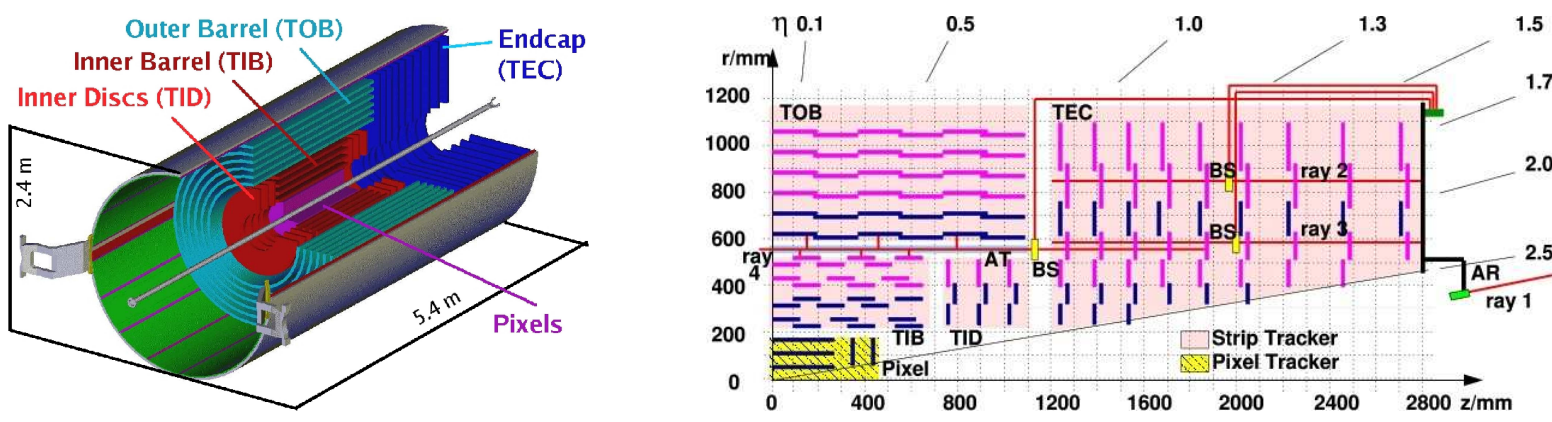

Figure 1: Tracker Layout (left) and Laser Alignment System Layout (right)

\section{CMS Tracker Alignment Strategy}

The strategy of CMS tracker alignment incorporates all available information - tracks, optical survey information, and the laser alignment system (LAS). Track-based alignment is absolutely necessary to achieve a sensor precision on the order of a few microns. The goal in track based alignment is to minimize a global $\chi^{2}$, an example of which is given in Eq. 2.1.

$$
\chi^{2}=\sum_{i}^{\text {tracks }} r_{i}^{T}(\mathbf{p}, \mathbf{q}) V_{i}^{-1} r_{i}(\mathbf{p}, \mathbf{q})
$$

In this equation, $r$ is the residual, the difference between the track extrapolation and the hit; $V$ is the residual covariance matrix; and $\mathbf{p}$ and $\mathbf{q}$ are the sensor and track parameters, respectively. CMS tracker alignment employs three alignment algorithms: HIP (Hits and Impact Points) [3], a local 
iterative method; Kalman Filter Algorithm [4], a global method updating parameters after every track; and Millepede II [5], a global method using global matrix inversion.

The survey measurements of the tracker are done at various tracker hierarchy levels with various sets of techniques. In the PXB, PXF, TIB, and TID, survey is done at the module level and, in some cases, at some higher level structures. In the TOB and TEC, survey is only done at the level of higher structures. Further, work has been done to incorporate the survey measurements into the track-based alignment as an extra constraint. In Eq. 2.2, the formalism for how survey constraint is added into the track-based $\chi^{2}$ term is shown

$$
\chi^{2}=\sum_{i}^{\text {tracks }} r_{i}^{T}(\mathbf{p}, \mathbf{q}) V_{i}^{-1} r_{i}(\mathbf{p}, \mathbf{q})+\sum_{j}^{\text {survey }} r_{* j}^{T}(\mathbf{p}) V_{i}^{-1} r_{* j}(\mathbf{p})
$$

In this equation, $j$ runs over all tracker survey hierarchies.

The final input to the tracker alignment comes from the LAS [2]. The goal of the LAS is to provide continuous position measurements of large scale structures in the TIB, TOB, and TEC. The precision of the LAS is on the order of 100 microns standalone and 10 microns monitoring over time. The layout of the LAS is given in Fig. 1.

\section{Recent Alignment Activities}

In the following section of these proceedings, we discuss the most recent exercises by the CMS tracker alignment group.

\subsection{Tracker Alignment at the CSA08 with MC}

During May of 2008, CMS tracker alignment was also involved in CSA08 (Computing, Software, and Analysis 2008) which tested the full workflow of alignment in real time. It was a two week exercise where the first week corresponded to $1 \mathrm{pb}^{-1}$ of data and the second week corresponded to $10 \mathrm{pb}^{-1}$ of data. Alignment constants from week 1 were to be used in week 2 . The exercise was completed in time; data, configuration, and alignment workflow all ran smoothly. With respect to the starting geometry which simulated startup misalignment, overall track $\chi^{2}$ was dramatically improved. The RMS of the difference between the aligned and true geometries in the most sensitive degree of freedom was about $35 \mu \mathrm{m}$ over the entire tracker and about $3 \mu \mathrm{m}$ in the PXB. We should point out this was a MC exercise excluding systematics; however, the successful test of the full alignment workflow with positive results provided valuable experience.

\subsection{Tracker Alignment at the CMS Global Runs with Cosmic Ray Data}

In July and August of 2008, CMS collected about 600k muons originating from air-showers suitable for alignment with a nearly full CMS tracker at $0 \mathrm{~T}$ during its Global Runs. Most of the strip tracker was active and alignment was attempted at the sensor level. The pixel detector was only active for a short time and statistics were not enough to align, but regardless, it was a first experience with a globally integrated pixel detector. Two alignment algorithms were run, HIP and Millepede. In particular, HIP ran track-based alignment using survey constraint as outlined in Sec. 2 while Millepede ran a solely track-based alignment. Validation of both alignment algorithms showed that they yielded similar results while both improving overall track $\chi^{2}$ and hit residuals. 
As a further validation, we show in Fig. 2 the mean of the hit residuals per module for modules with at least 100 hits for the TOB. The advantage of such a validation is that it reduces the contribution to the residuals from multiple scattering, significant especially for zero field cosmics. In the figure, a comparison is made between the design geometry and aligned geometry validated over data and the design geometry validated over MC. We note the dramatic improvement between the designed and aligned geometries over data while the presence of the design geometry over MC gives a measure of the statistical contribution. Also in Fig. 2, the impact of alignment on tracking
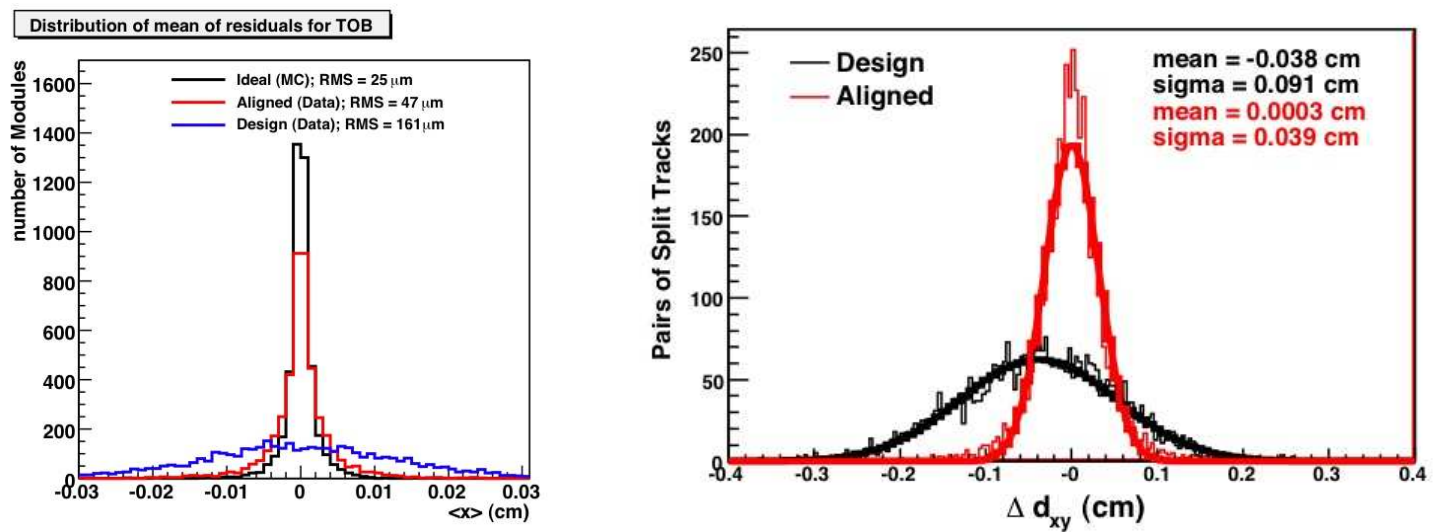

Figure 2: Distribution of the mean of residuals for TOB sensors with more than 100 hits (left) and Impact parameter, $d_{0}\left(d_{x y}\right)$, resolution for top vs. bottom cosmic tracks (right)

is given. In this validation, a single cosmic track is split along the point of closest approach to the beamline and compared as individual tracks. This gives a measure of the track parameters' bias and resolution. We see an improvement from the designed to aligned geometry in $d_{0}$ (pictured), $d_{z}$, $\phi$, and $\theta$. Overall, tracker alignment during CMS Global Runs at zero field successfully exercised the full workflow providing dramatic improvement.

\section{Implication of Tracker Alignment on Physics Performace}

While CMS tracker alignment has been involved in analyses both with simulation and cosmic data, a parallel analysis has been done to understand the impact of tracker alignment on physics performance. To understand the effect of misalignment, a number of misalignment scenarios were created: 'SurveyLAS', 'SurveyLASCosmics', '10 $p b^{-1}$ ', and '100 $p b^{-1}$. These misalignments each contain a presumed alignment precision. For example in 'SurveyLASCosmics', this is the presumed level of alignment precision using survey, LAS and cosmic data. In the integrated luminosity scenarios, this is the presumed alignment precision after that amount of data is collected. All of these scenarios are laid out in greater detail here [6].

With these scenarios, we look at the effect of misalignment on tracking. In particular, the alignment position errors (APEs), errors assigned based on the level of misalignment, factor greatly into tracking. This can be seen in Fig. 3. The track finding efficiency remains high if the APEs agree well with the given misalignment regardless of the scenario. However, when the APEs are set 
to zero, track finding efficiency suffers greatly. Further, we find that impact parameter resolution,
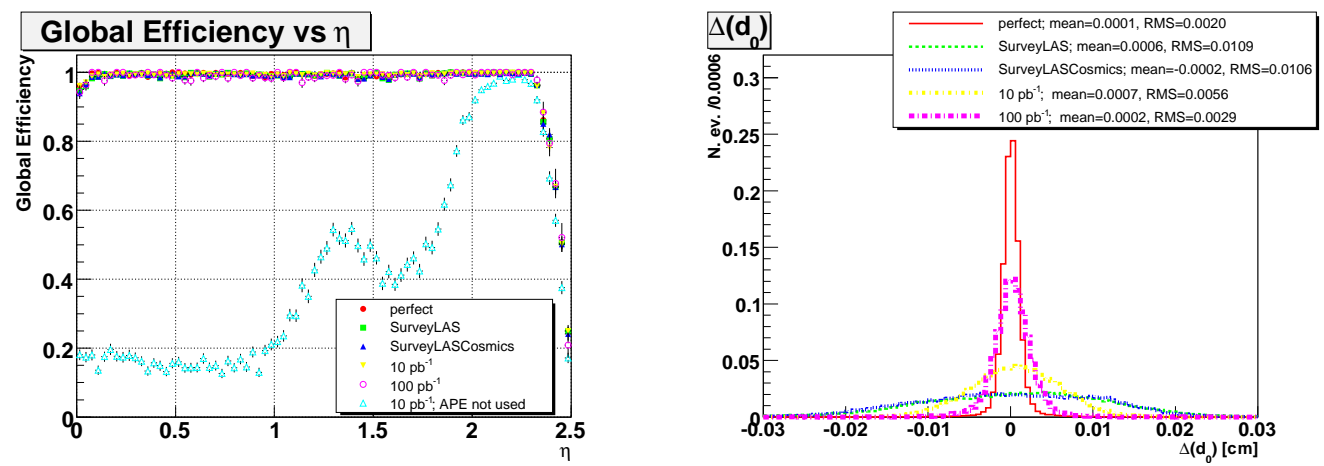

Figure 3: Track finding efficiency (left) and impact parameter, $d_{0}$ (right), for various misalignment scenarios.

$d_{0}$ and $d_{z}$, are greatly affected by misalignment of the PXB. Fig. 3 reflects the impact in $d_{0}$. In scenarios in which the PXB are greatly misaligned ('SurveyLAS' and 'SurveyLASCosmics'), the impact parameter resolution suffers greatly. APE estimation and PXB alignment are two important factors impacting tracking performance. Further details and analysis are presented in [6].

\section{Summary}

Within these proceedings, we give a summary of the current status of CMS tracker alignment. Initially, the basic strategy of CMS tracker alignment was discussed. Then, an overview of the various recent analyses was presented with encouraging and positive results. Finally, the impact of alignment on physics performance was discussed where it was determined that APE estimation and PXB alignment are vital issues. All such studies have been an invaluable experience for CMS tracker alignment, and our recent progress is encouraging for LHC startup.

\section{References}

[1] CMS Physics Technical Design Report, Volume I: Detector Performance and Software, CERN/LHCC 2006-001, CMS TDR 8.1

[2] A. Ostapchouk et al., "The Alignment System of the CMS Tracker", CMS NOTE-2001/053

[3] V. Karimaki, T. Lampen, F.-P. Schilling, "The HIP Algorithm for Track Based Alignment and its Application to the CMS Pixel Detector," CMS NOTE-2006/018

[4] E. Widl, R. Frühwirth W. Adam, "A Kalman Filter for Track-based Alignment", CMS NOTE-2006/022

[5] G. Flucke, P. Schleper, G. Steinbrück, M. Stoye, "A Study of Full Scale CMS Tracker Alignment using High Momentum Muons and Cosmics", CMS NOTE-2008/008

[6] T. Lampen, N. Filippis, F.-P. Schilling, A. Schmidt, M. Weber, "Comprehensive Set of Misalignment Scenarios for the CMS Tracker," CMS NOTE2008/029 